\title{
Mixing Phenomena in a Liquid Bath Stirred by Gas Jets through Side and Inclined Nozzles
}

\author{
Sergey V. KOMAROV, Kenji ITOH, ${ }^{1)}$ Masamichi SANO ${ }^{2)}$ and Konstantin A. BLINOV ${ }^{3)}$
}

Formerly Department of Materials Processing Engineering, Nagoya University. Now at Russia, Moscow, Steel and Alloys Institute, Leninskiy Avenue, Moscow, Russia. 1 1) Formerly Graduate School, Nagoya University. Now at Kobe Steel, Ltd., Nadahamahigashi-cho, Nada-ku, Kobe, Hyogo-ken, 657 Japan. 2) Department of Materials Processing Engineering, Faculty of Engineering, Nagoya University, Furo-cho, Chikusa-ku, Nagoya, Aichi-ken, 461-01 Japan.

3) A. A. Baykov Institute of Metallurgy, Russia Academy of Sciences, Leninskiy Avenue, Moscow, Russia.

(Received on December 2, 1992; accepted in final form on March 19, 1993)

\begin{abstract}
The perfect mixing time, $t_{m}$ of the water bath, during gas injection through bottom (vertical and iclined) and side nozzles has been measured by using electrical conductivity technique. Effects of gas flow rate, bath depth, nozzle angle and location have been examined. The measurements indicate that under the condition of a shallow bath $\left(H / D=0.31\right.$ ) and large gas flow rate (specific power $\varepsilon>10^{-1} \mathrm{~W} / \mathrm{kg}$ ) influences of the nozzle angle and nozzle location on the mixing time are significant. On the other hand, effects of those parameters on $t_{m}$ are negligible in a deep bath $(H / D=1.25)$. Change in the tracer concentration with time was predicted by numerical modelling. A satisfactory agreement between the computed and experimental results can be obtained by parameter fitting for the average plume rise velocity and effective viscosity in the mathematical model. It is presumed that turbulent mass transfer of the tracer is dominant in the deep bath, while the convection of liquid is more effective in the homogenization in the shallow bath.
\end{abstract}

KEY WORDS: perfect mixing time; cold model; side and inclined injection; numerical modelling; turbulent mass transfer; convection.

\section{Introduction}

Mass transfer phenomena in metallurgical processes are largely governed by hydrodynamical characteristics of a melt bath. In essence, hydrodynamics together with surface phenomena has a dominant effect on the refining rate in the processes. Therefore, our understanding of hydrodynamical phenomena is very important to solve many practical problems.

Recently, new metallurgical processes have been developed to refine metal in a liquid state by means of gas injection. These processes have found application both at the pretreatment and at the ladle treatment stages. The injection produces circulation and agitation in the bath, which are mainly connected with two effects - convection and turbulent diffusion. It is well known that the mixing intensity depends on a gas flow rate. $^{1,3}$ Besides tuyere location at the bottom, bath depth also has an effect. Many studies on the mixing phenomena have been carried out by using numerical modelling. ${ }^{4,5)}$ It is found that fluid flow velocity and turbulence energy distributions in the stirred bath are nonuniform. This leads to the appearance of temperature and concentration distributions in the bath. These results have been confirmed by experiments. ${ }^{6 \text { ) }}$

The purpose of the present work is to study the mixing phenomena in a gas stirred liquid bath. The liquid bath is stirred by inclined gas injection at the bottom or side gas blowing. The effects of direction of gas flow and tuyere location on mixing time are studied. A numerical calculation is made to clarify the dispersion characteristics of added tracer.

\section{Experimental}

The experimental set is shown in Fig. 1. The vessel made of acrylic resin has an inner diameter of $480 \mathrm{~mm}$

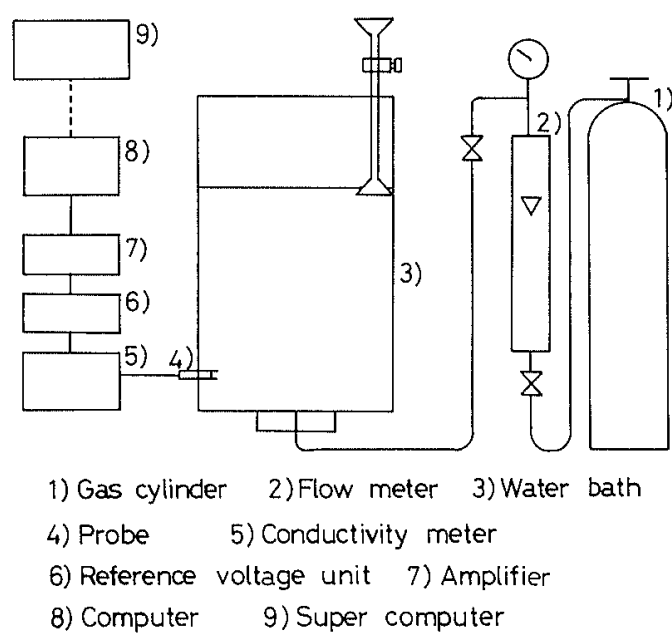

Fig. 1. Experimental apparatus. 
and a height of $700 \mathrm{~mm}$. Nitrogen was injected into the bath through one or four nozzles. In the case of bottom blowing, the nozzles made of stainless steel were located at the replaceable bottom. The four nozzles were placed along the circle with various radiuses at angles of 90 , 60,45 and $30^{\circ}$ to the bottom plate, as shown in Fig. 2. When the number of nozzle is one, it was placed at the bottom center. A series of experiments have been made for mixing phenomena in side blowing. The side nozzles were installed in such a way that extensions of their axes formed a square on a horizontal section of the vessel (Fig. 2). The distance between the bottom and the side nozzle was $70 \mathrm{~mm}$. The inner and outer diameters of the nozzle were 1 and $3 \mathrm{~mm}$.

A platinum electrical conductivity probe was placed at a height of $60 \mathrm{~mm}$ from the bottom. The output signal of electrical conductivity meter was recorded by a personal computer combined with $A / D$ interface and an amplifier. To compensate for an increase in the output signal, a reference voltage unit was set in the electrical circuit. The $\mathrm{KCl}$ solution $\left(2 \mathrm{kmol} / \mathrm{m}^{3}\right)$ was used as a tracer. To minimize the influence of the initial condition owing to tracer addition and wave motion at the bath surface, a funnel was used. It was submerged in the bath by a depth of $10 \mathrm{~mm}$ at a position being most distant from the electrical conductivity probe. Predetermined amount of the tracer solution was added to the water bath.

Before starting measurements, nitrogen was blown into the bath for $3-5 \mathrm{~min}$ to ensure the steady flow in

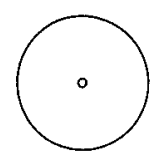

A

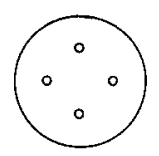

C

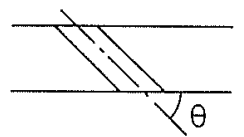

bottom injection
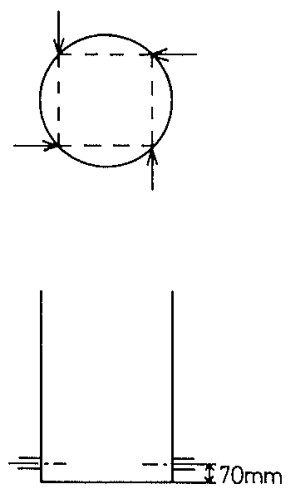

side injection
Fig. 2. Location and inclination of bottom and side nozzles. the vessel. Then, for determination of the electrical noise, voltage fluctuations were checked. As a criterion for determination of the mixing time in the stirred liquid bath, $2.5 \%$ relative deviation of the output signal from the steady-state value was adopted. When the electrical noise exceeded the relative deviation, the experiment was repeated until the noise came into the range of that value. The experimental conditions are summarized in Table 1.

\section{Results and Discussion}

All experimental results of homogenization of added tracer can be divided into three types as it is indicated in Fig. 3. The curves in Fig. 3 show the changes in output of electrical conductivity with time. The output signal of the first type (a) is monotonically increased to the steady state value without taking into account the
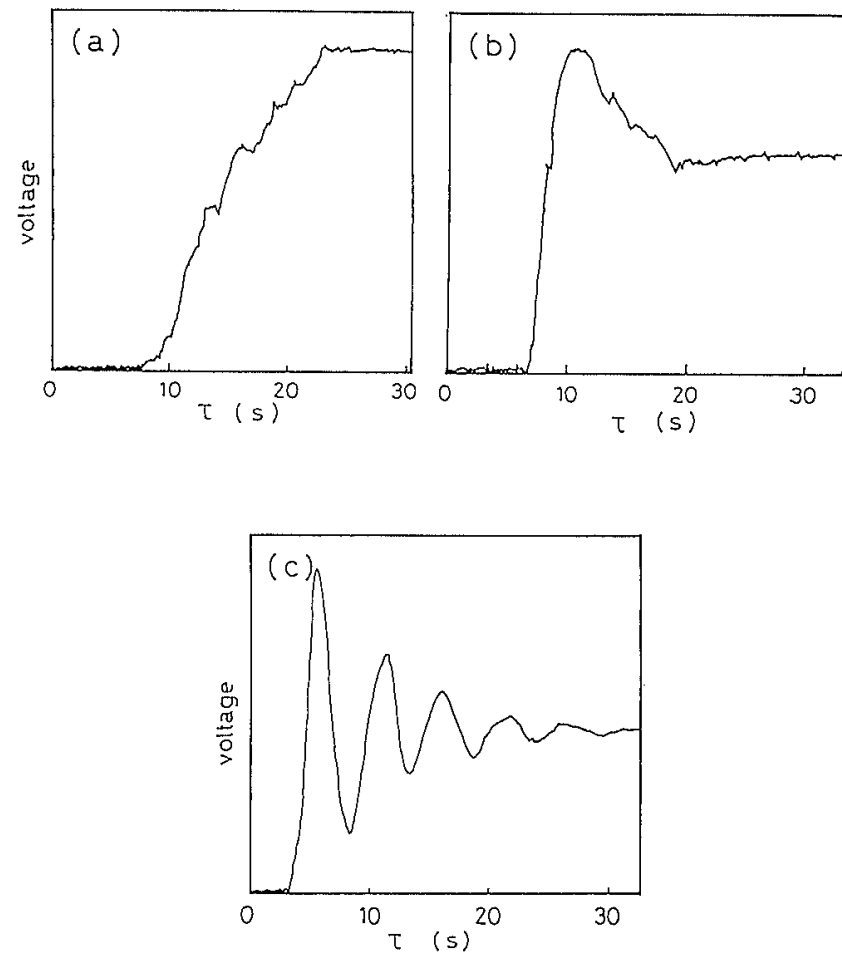

\begin{tabular}{lllll}
\hline & $Q\left(\mathrm{Nm}^{3} / \mathrm{s}\right)$ & $H(\mathrm{~m})$ & $N_{t}$ & $\theta(\mathrm{deg})$ \\
\hline a & $3.3 \times 10^{-4}$ & 0.6 & 1 & 90 \\
b & $6.7 \times 10^{-4}$ & 0.4 & 4 & 60 \\
c & $1.0 \times 10^{-3}$ & 0.15 & 4 & 30 \\
\hline
\end{tabular}

Fig. 3. Typical pattern of change in output signal with time.

Table 1. Experimental variables.

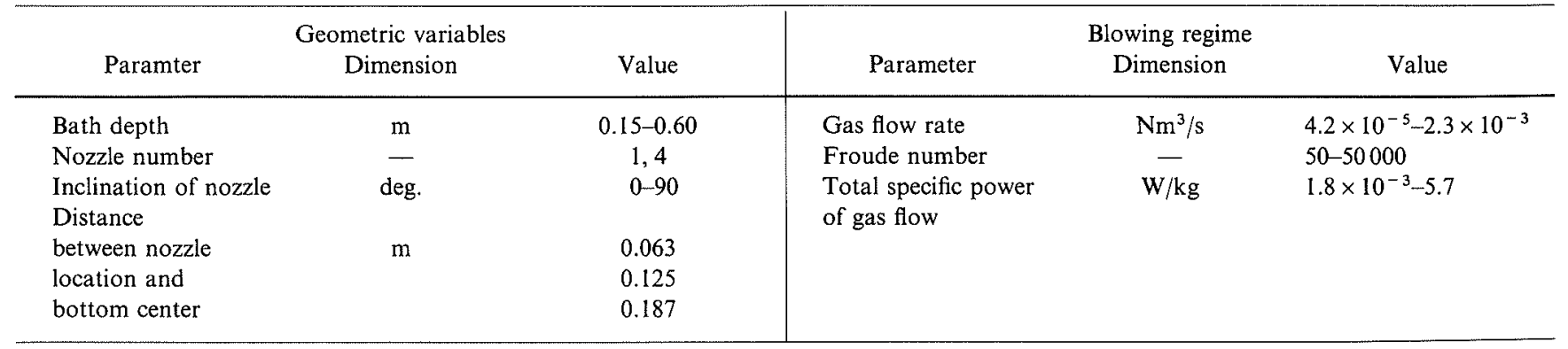

Froude number $F r=\rho_{g} u_{0}^{2} /\left(\rho_{l} g d_{n}\right)$. 
small-scale signal fluctuations. The curve of the second type (b) has one concentration maximum before reaching the steady value. The third type (c) goes through a few maxima and minima damping with time. It is found that transition in signal type from the first to the third one takes place, as either the bath depth or nozzle inclination is decreased or the gas flow rate is increased.

Applicability of the cold model results to industrial equipments depends on the choice of similarity criteria. In current investigations, the Froude number as well as Newton number ${ }^{7)}$ are widely used. Nevertheless there are reasons to presume that some important physical phenomena occurring at the energetic gas injection into a liquid bath are not taken into account by these criteria. As it follows from previous studies, ${ }^{3,8}$ the inertial force of injected gas plays an important role at the initial zone near the nozzle (jet region), while the buoyancy force governs the behaviour of the plume in the zone distant from nozzle (bubble region). However, in both of these regions, the momentum transfer from gas to liquid phase is determined by the turbulent viscous force. Because the physics of the phenomena is very complicated and is not clear yet up to date, the criterion including the turbulent viscous force can not be expressed by a simple mathematical relationship. Therefore, as a similarity criterion the total power of the gas being injected into the bath per liquid mass is considered as reasonable. Specific powers of the injected gas in the jet region $\varepsilon_{j}$ and in the bubble region $\varepsilon_{b}$ can be written by the following equation: ${ }^{1)}$

$$
\begin{gathered}
\varepsilon_{j}=\frac{\rho_{g} u_{0}^{2} Q}{2 W} \\
\varepsilon_{b}=\frac{Q R T}{V_{M} W} \ln \frac{P_{1}}{P_{2}}
\end{gathered}
$$

Thus hydrodynamical processes in the cold model and the practical equipment are assumed to be similar if the variables, such as $\varepsilon\left(=\varepsilon_{b}+k \varepsilon_{j}\right), H / D, N_{t}, L / D$ and $\theta$, take the same values in both the systems, where the coefficient $k$ means physically the part of the kinetic energy which is transferred from gas to liquid bath.

In the present investigation, $k$ was taken as $0.15{ }^{2}{ }^{2}$ Results presented in Figs. 4(a) and 4(b) show the relationship between the mixing time $t_{m}$ and the specific power of gas $\varepsilon$ plotted in logarithmic coordinates for conditions of $N_{t}=1, \theta=90^{\circ}$ (a: bottom blowing) and $N_{t}=4$ (b: side blowing). To plot the lines, the well known empirical relationship between $t_{m}$ and $\varepsilon\left(t_{m}=A \varepsilon^{q}\right)$ is used. Calculation of $A$ and $q$ was carried out by means of the least square method. Total discrepancy $F$ between calculated and experimental mixing times, $t_{m, o}$ and $t_{m, e}$, is expressed by the Eq. (3):

$$
F=\sum_{i=1}^{n}\left(t_{m i, o}-t_{m i, e}\right)^{2}
$$

The experimental results can be correlated by two straight lines in the logarithmic coordinates. A cross point of these lines is so determined that the discrepancy, $F$, is minimized.

It has been found that the tendency of decrease in $t_{m}$
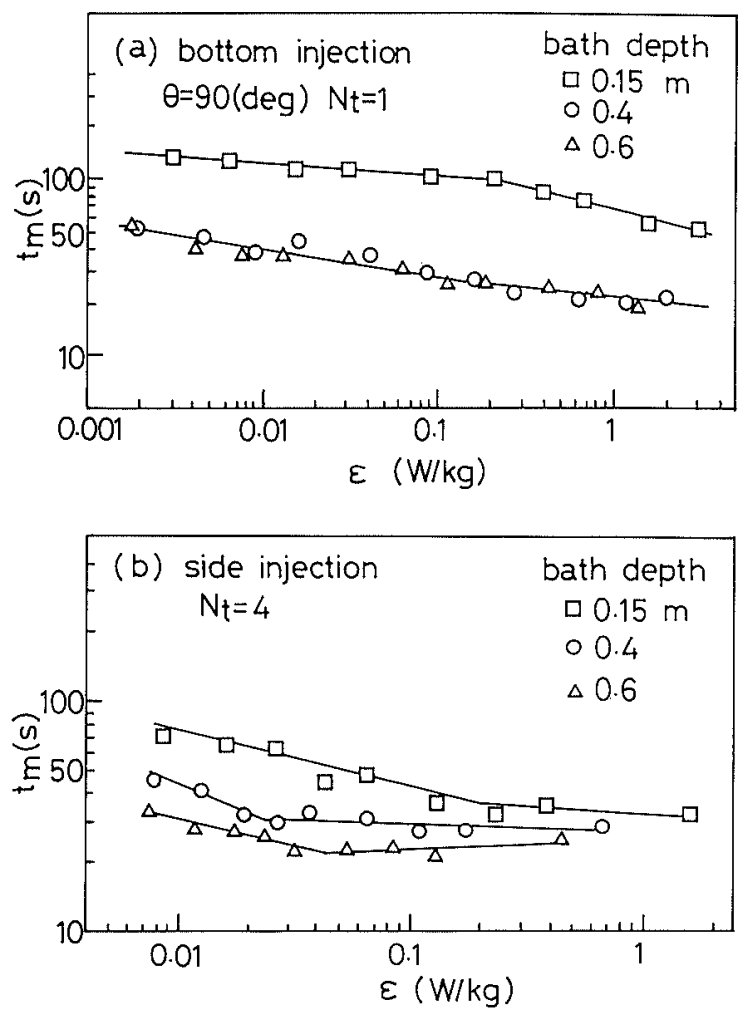

Fig. 4. Effect of specific power on mixing time.
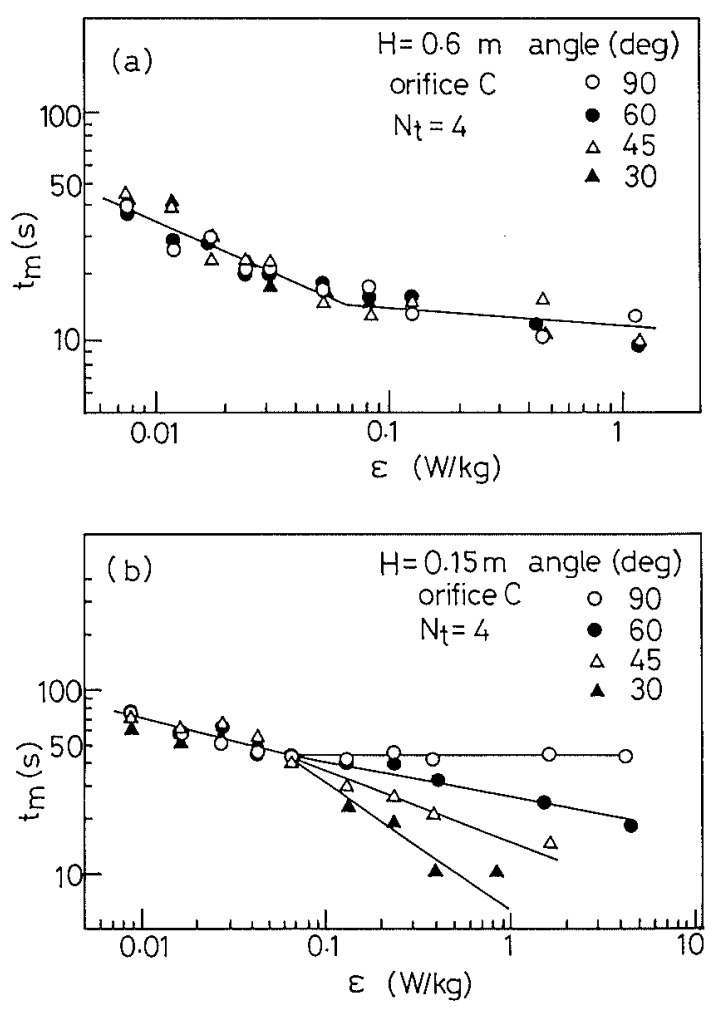

Fig. 5. Effect of specific power on mixing time.

is observed with increasing bath depth. Influence of the specific power on the mixing time depends on the bath depth and the nozzle angle. The nozzle angle has a significant influence in the case of a shallow bath (Fig. 5(b)); at $\varepsilon$ larger than $10^{-1} \mathrm{~W} / \mathrm{kg}$ the smaller the angle is, the less is the mixing time, while in the range of $\varepsilon<10^{-1} \mathrm{~W} / \mathrm{kg}$, the mixing time is independent of the 
nozzle angle. For instance, the difference between $t_{m}$ for $\theta=90$ and $30^{\circ}$ reaches up to five times at a high gas flow rate. However, the influence of the nozzle angle on the mixing time was within experimental error for a deep bath (Fig. 5(a)).

Results in Figs. 6 and 7 indicate influences of the

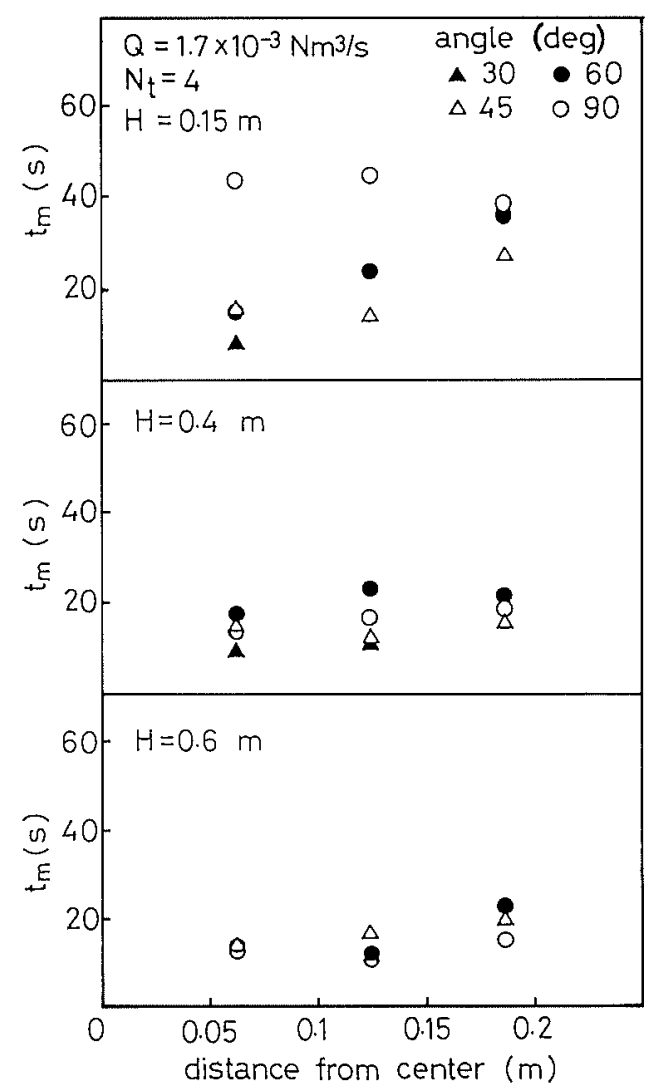

Fig. 6. Effect of nozzle location on mixing time.

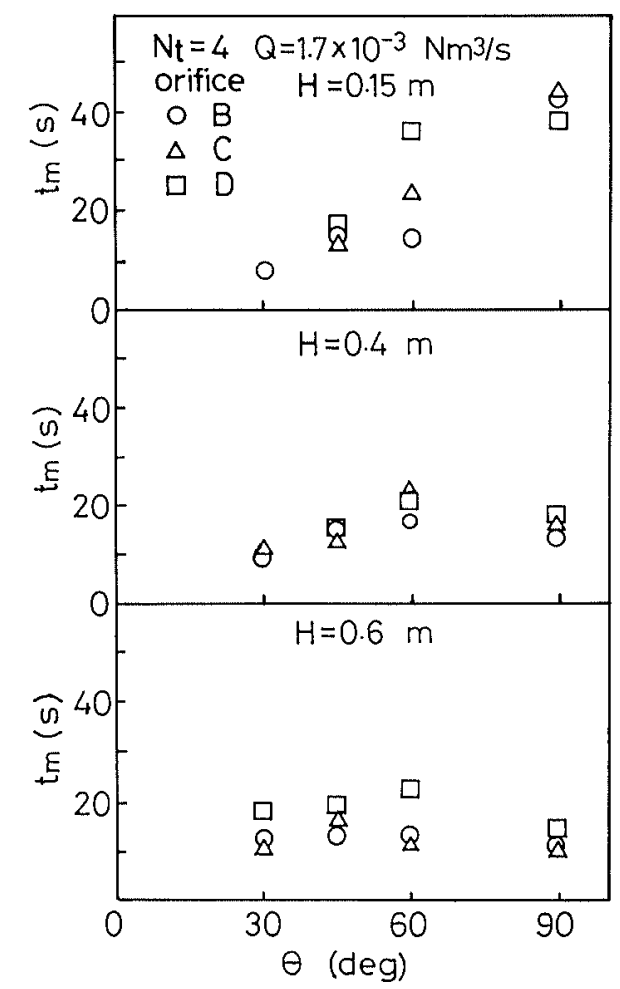

Fig. 7. Effect of nozzle inclination on mixing time. nozzle location and the nozzle angle on the mixing time. Their influences are dependent on the bath depth, but on the whole, decreased as the bath depth is increased.

\subsection{Mixing Time}

The purpose of a mathematical model presented here is to show influences of such parameters as turbulent viscosity, plume rise velocity, and bath depth on the mixing time in a liquid bath stirred by gas injection. The following principal assumptions are made:

1) Cylindrical symmetry,

2) Steady state conditions and constant density in the flow field,

3) Description of liquid velocity in the plume by the average plume rise velocity $v_{p}$ in the vertical $z$ coordinate, and

4) Isotropic homogeneous turbulence in the liquid. The mathematical model can be formulated as follows

$$
\begin{aligned}
& \rho_{l} u \frac{\partial u}{\partial r}+\rho_{l} v \frac{\partial u}{\partial z}=-\frac{\partial P}{\partial r}+\mu_{l} \nabla^{2} u-\rho_{l} \frac{u^{2}}{r} \\
& \rho_{l} u \frac{\partial v}{\partial r}+\rho_{l} v \frac{\partial v}{\partial z}=-\frac{\partial P}{\partial z}+\mu_{l} \nabla^{2} v \\
& \frac{\partial u}{\partial r}+\frac{\partial v}{\partial z}+\frac{u}{r}=0 \\
& \frac{\partial C}{\partial \tau}+u \frac{\partial C}{\partial r}+v \frac{\partial C}{\partial z}=D_{l} \nabla^{2} C \\
& \iint C r d r d z=\text { const. }
\end{aligned}
$$

where,

$$
\nabla^{2}=\frac{\partial^{2}}{\partial r^{2}}+\frac{\partial^{2}}{\partial z^{2}}+\frac{1}{r} \frac{\partial}{\partial r} \quad \text { (Laplace operator) }
$$

The model involves Navier-Stokes Eqs. (4) and (5), the continuity Eq. (6), the diffusion Eq. (7) and the mass conservation Eq. (8). Equations (4) to (9) are solved under the following initial and boundary conditions:

The boundary conditions:

$$
\begin{aligned}
& \text { 1. } z=0,0 \leqq r \leqq R: u=v=0, \frac{\partial C}{\partial z}=0 \\
& \text { 2. } r=R, 0 \leqq z \leqq H: u=v=0, \frac{\partial C}{\partial r}=0 \\
& \text { 3. } z=H, 0 \leqq r \leqq R: v=\frac{\partial v}{\partial z}=0, \frac{\partial C}{\partial z}=0 \\
& \text { 4. } r=0,0 \leqq z \leqq H: u=\frac{\partial v}{\partial r}=0, v=v_{p}, \frac{\partial C}{\partial r}=0
\end{aligned}
$$

The initial conditions $(\tau=0)$ :

5. $H-h \leqq z \leqq H, 0 \leqq r \leqq R^{\prime}: C=C_{0}$

$$
\begin{aligned}
& H-h \leqq z \leqq H, R^{\prime}<r \leqq R: C=0 \text { and } \\
& 0 \leqq z<H-h, 0 \leqq r \leqq R: C=0
\end{aligned}
$$




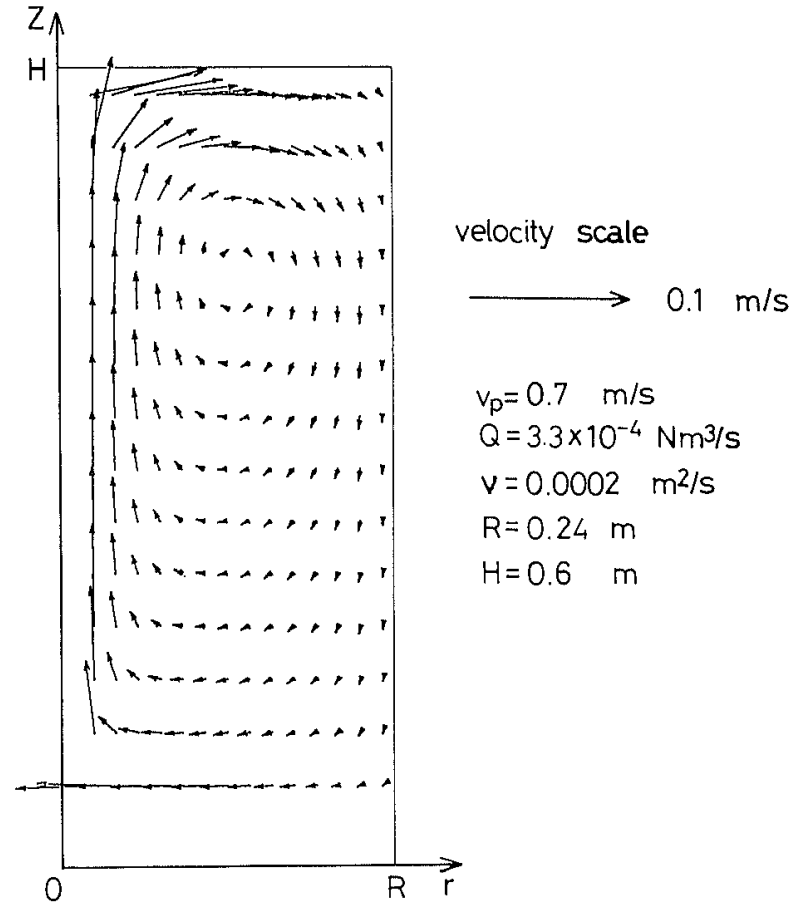

Fig. 8. Calculated fluid flow pattern.

It is assumed that the tracer is distributed initially in the bounded space of the bath (Eq. (14)). It is necessary to note that the present mathematical model can not be applied to simulate the liquid flow for any distributed and inclined gas blowing. Thus all calculated results are concerned with gas injection through one nozzle located at the bottom center.

The Eqs. (4) to (8) were solved using a $20 \times 20$ uniform grid structure by Gauss-Seidel method with successive overrelaxation. ${ }^{11)}$

\subsection{Mechanism of Homogenization in the Bath}

Figure 8 depicts the flow pattern in the vessel for bath depth $0.6 \mathrm{~m}$ and gas flow rate $3.3 \times 10^{-4} \mathrm{Nm}^{3} / \mathrm{s}$. All calculations using this type of model require prior knowledge of the effective viscosity $\mu_{l}$ and the average plume rise velocity $v_{p}$. In the present work they are determined by minimizing the discrepancy between the calculated and experimental results of change in tracer concentration with time.

Figure 8 shows that the liquid flow rate is relatively high near the free surface. A stagnant zone is found at a distance of half-radius from the vessel axis.

It is well known that the mixing in a liquid bath is mainly connected with two phenomena-convection and turbulence mass transfer. The convection is affected by the flow of a large-scale liquid volume, while the turbulence mass transfer depends on the turbulent diffusivity in the liquid bath. Geometric parameters of the vessel have an influence on both of them. The relationships which have been published in the literature permit one estimating both the effective viscosity and the average plume rise velocity depending on the gas flow rate (dynamic factor) as well as on the bath depth and diameter (geometric factors). Review of the available expressions has been done in the work. ${ }^{9}$ ) Equations (15) and (16) have been obtained on the basis
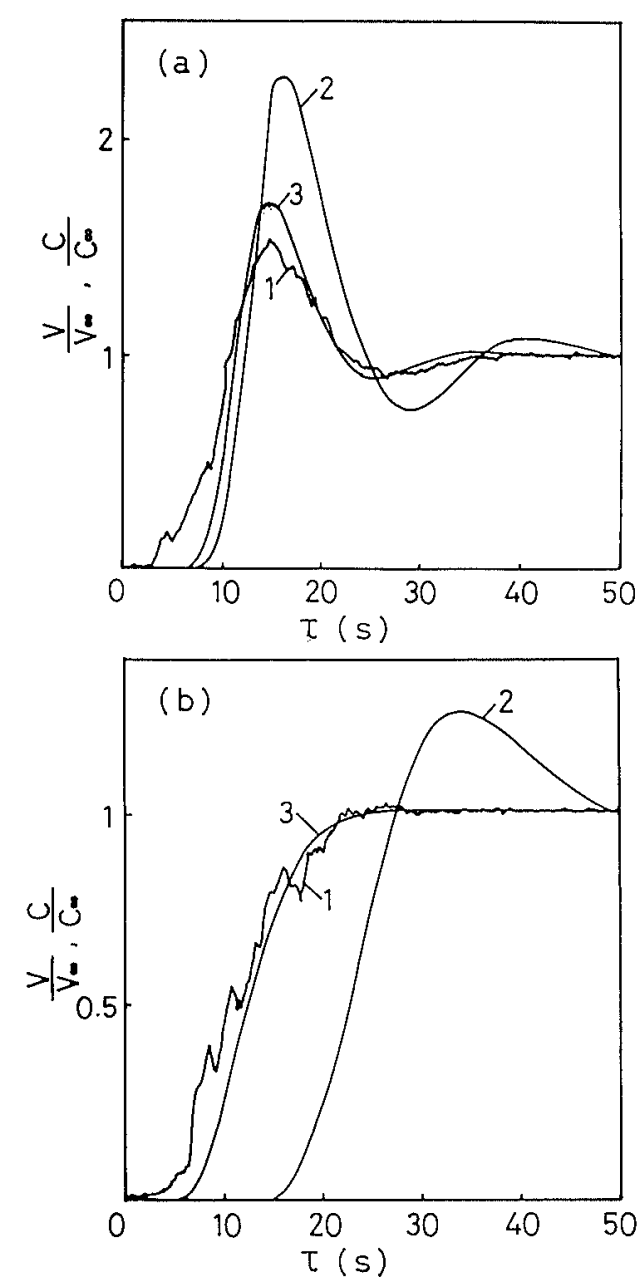

\begin{tabular}{lllll}
\hline & $v_{p}(\mathrm{~m} / \mathrm{s})$ & $v\left(\mathrm{~m}^{2} / \mathrm{s}\right)$ & $H(\mathrm{~m})$ & $Q\left(\mathrm{Nm}^{3} / \mathrm{s}\right)$ \\
\hline $\mathrm{a}$ & 0.55 & $4.2 \times 10^{-5}$ & 0.15 & $8.3 \times 10^{-4}$ \\
$\mathrm{~b}$ & 0.80 & $1.5 \times 10^{-4}$ & 0.4 & $1.0 \times 10^{-3}$ \\
\hline
\end{tabular}

Fig. 9. Change in relative voltage (I) and tracer concentration $(2,3)$ with time. 1 - experiment, 2 -calculation by using Eqs. (15) and (16), 3-calculation by fitting $v$ and $v_{p}$.

of the assumption of isotropic homogeneous turbulence and by using the empirical correlation to determine the proportionality factor.

$$
\begin{gathered}
\mu_{l}=4.9 \times 10^{-4} \rho_{l} H(Q g / D)^{1 / 3} \\
v_{p}=4.78 Q^{1 / 3}(H / 2 D)^{1 / 4} \ldots \ldots
\end{gathered}
$$

The curves in Figs. 9 (a) and 9(b) show comparison of the experimental result (1) with the numerical modelling result ( 2 and 3 ). The curve 2 is obtained by using Eqs. (15) and (16). It is seen that the curve 2 does not agree with the experimental result. The curve 3 depicts the result obtained by optimizing the effective viscosity and plume rise velocity so that agreement is obtained between the calculation and the experiment. In the calculation, the turbulent diffusivity is assumed to be equal to the turbulent kinematic viscosity. As a criterion of the optimization, the relative discrepancy in the tracer concentration between calculated and experimental values was taken. The agreement was considered to be satisfactory if the average relative discrepancy was 

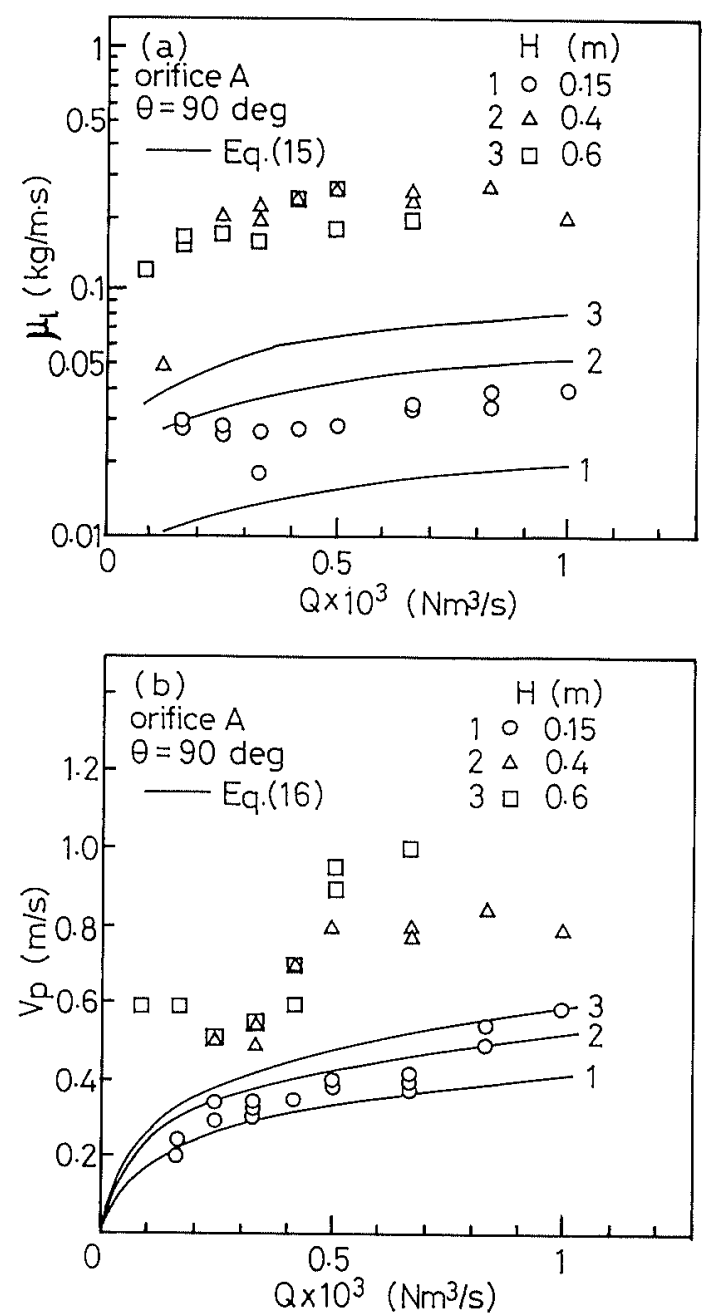

Fig. 10. Effect of gas flow rate on effective viscosity $\mu_{t}$ and average plume rise velocity $\left(v_{p}\right)$.

not over $25 \%$. Here, the relative discrepancy, $E$, is defined as

$$
E=\frac{X_{e}(\tau)-X_{c}(\tau)}{X_{e}(\tau)}
$$

and the average relative discrepancy, $E_{a v}$, as

$$
E_{a v}=\frac{\int_{0}^{\tau} \frac{X_{e}(\tau)-X_{c}(\tau)}{X_{e}(\tau)} d \tau}{\int_{0}^{\tau} d \tau}
$$

where, $X_{e}(\tau)=V / V_{\infty}, X_{c}(\tau)=C / C_{\infty}, V$ is the voltage measured by the conductivity meter, $C$ is the calculated tracer concentration, and the subscript $\infty$ denotes the equilibrium value. The program algorithm was constructed in such a way that the relative steady-state output signal of the electrical conductivity meter and the final calculated relative tracer concentration became equal.

In Fig. 10 the values of $\mu_{l}$ and $v_{p}$ found as a result of the optimization are compared with those calculated from Eqs. (15) and (16). The discrepancy between average plume rise velocities obtained by the model and by using Eq. (16) is larger at higher gas flow rate. The effective viscosity of this study is larger than that given
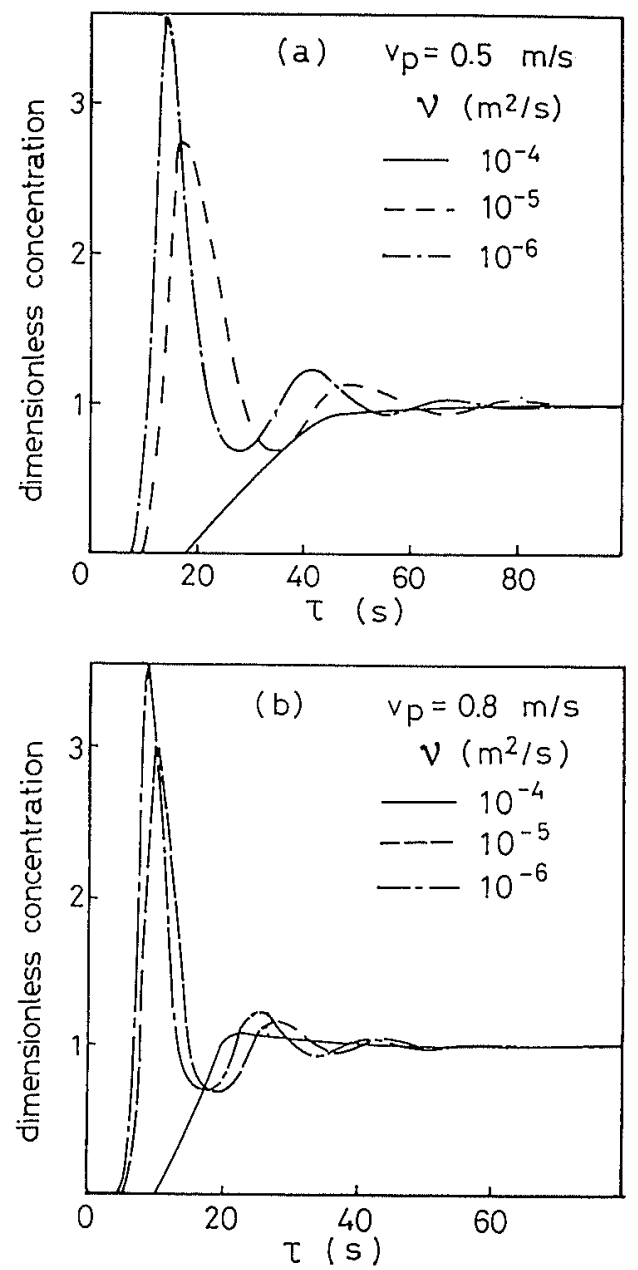

Fig. 11. Influence of effective kinematic viscosity on change in relative tracer concentration with time.

by Eq. (15) in the whole range of gas flow rate. In general, the discrepancy in $\mu_{l}$ is greater than that in $v_{p}$.

It should be noted here that our purpose is not to develop a unique mathematical model for calculation of the mixing time under various conditions. But, the model is applied to interpret the experimental results of homogenization of added tracer from the view point of the mechanism of mixing phenomena. It is shown that the pattern of the tracer concentration change with time is greatly affected by the effective viscosity as well as the average plume rise velocity. This is indicated in Figs. 11(a) and 11(b). The curves show the relative tracer concentration change with time at the point of the probe location. It can be seen that when $\mu_{l}$ is increased and the velocity $v_{p}$ is kept constant, the curve shape changes from a monotonically increase type to an oscillating type. Figure 11 shows that the higher the plume rise velocity the sharper the peak in the oscillating curve.

Thus, the difference between the prime results shown in Fig. 3 is due to the different effective viscosity and plume rise velocity $v_{p}$ in the bath. Eqs. (15) and (16) show that $\mu_{l}$ is proportional to the bath depth while the velocity $v_{p}$ is proportional to fourth root of the depth. Therefore, because of large effective viscosity in the deep bath, homogenization of tracer distribution proceeds through the turbulent mass transfer for which the 
monotonic change in the tracer concentration with time is characterized. On the other hand, convective homogenization should be expected to be dominant in the shallow bath because of the low turbulence level. As a result, the tracer concentration change with time takes an oscillating pattern.

The tracer concentration change with time depends on at least three factors: measurement point in the liquid bath, effective viscosity and average plume rise velocity. The influence of probe location on the mixing time has been studied. ${ }^{10}$ ) It was shown that both the mixing time and the curve shape depend on the probe location. This comes from the fact that the nature of the mixing phenomena at each point is governed not only by the average dynamic parameters but also by their local values.

The turbulent viscosity by the nature is a tensor value and, therefore, depends on the direction. However, the influence of the direction disappears with an increase in the gas flow rate in the case of a bounded volume like a gas stirred liquid bath. The liquid bath reaches the state named the isotropic homogeneous turbulence and can be described by the effective viscosity. Hence the nozzle location and nozzle inclination have no influence on the mass transfer in a liquid bath in the isotropic homogeneous turbulence state.
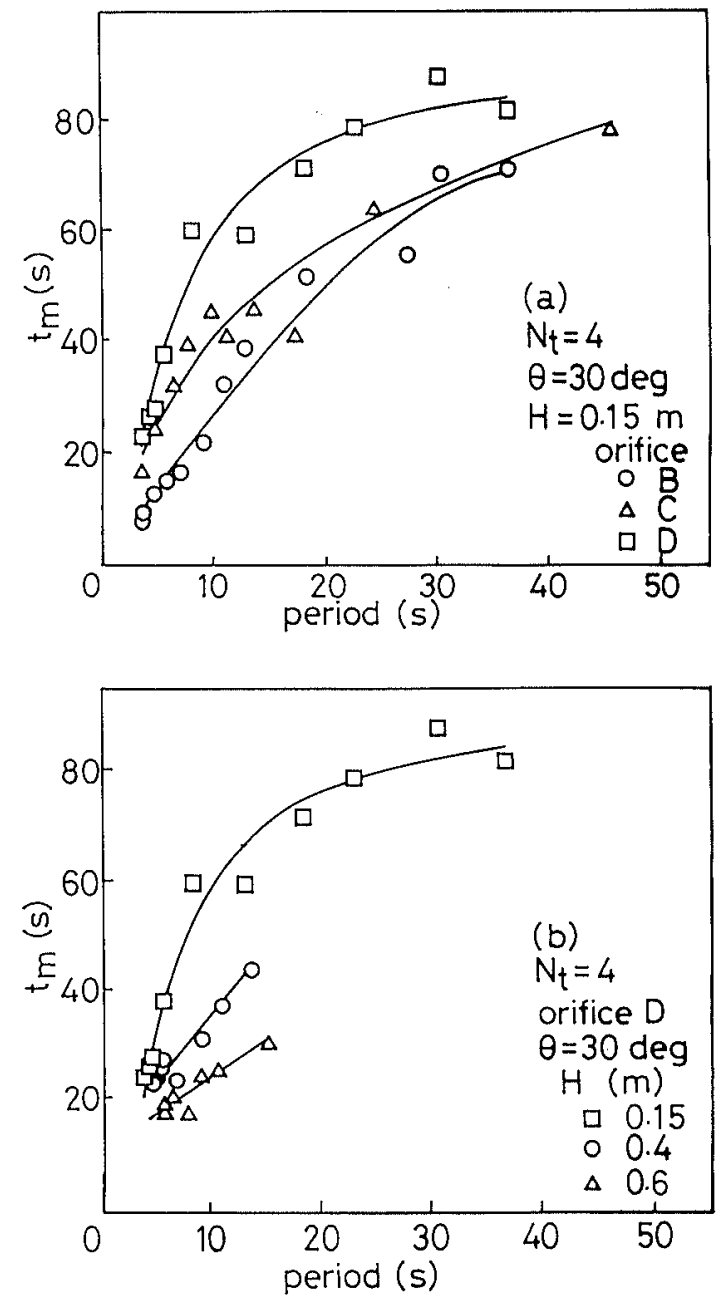

Fig. 12. Relationship between mixing time and apparent period of liquid circulation in the bath.
As shown in Figs. 4 and 5 , dependence of $t_{m}$ on specific power $\varepsilon$ changes at a certain value of $\varepsilon$. The reason why this point exists is not clear. Any change was not observed in the interaction between the gas flow and liquid bath excepting the large-scale rotating oscillations occurring in the bath at higher gas flow rate. These oscillations may have an effect on the fluid flow pattern and energy redistribution in the liquid.

On the other hand, in the case of a shallow bath (Fig. $5 b)$, the change in nozzle inclination and location has a significant influence on the liquid flow pattern. The inclined gas injection induces liquid rotation in the bath. The liquid is stirred in both the vertical and the horizontal directions. It has been found that the rotation becomes more intensive with an increase in the gas flow rate and decrease of the nozzle inclination. The relationship between the mixing time $t_{m}$ and period $t_{o}$ of the liquid circulation in the bath is shown in Figs. 12(a) and 12(b). The period was determined by the Fourier analysis of the oscillating output signal. Therefore, it would be more accurate to name it the apparent period of the liquid circulation. The gas injection through the bottom gives a clear relation between $t_{m}$ and $t_{o}$, which is dependent on the nozzle location.

As a final note, the nozzle location and nozzle inclination have much less effect on the mixing time in a deep bath, like a steel ladle, than it does in a shallow bath such as in a converter. In the former, the mixing is expected to be intensified by raising the turbulence level in the bath. On the other hand, the mass transfer and bath mixing in the latter can be essentially improved by injecting gas from the inclined nozzles.

\section{Conclusion}

Increase in rate of mass transfer in gas stirred bath can not be achieved without considering both the effects of geometric parameters of the vessel (bath depth and diameter) and dynamic parameter (in the present work-specific power of gas flow). According to the results of this study, the mechanism of mixing phenomena changes depending on the bath depth, gas flow rate and other factors. Homogenization in a shallow bath is governed by convection of liquid, while turbulent mass transfer is dominant in the homogenization in a deep bath.

As one of the techniques for mass transfer intensification, the bottom and the side gas injections have been studied. It is shown, that the nozzle inclination and location have influences of the mixing time depending on the bath depth and the gas flow rate. The effect of nozzle inclination on $t_{m}$ is more significant for the shallow bath $(H=0.15 \mathrm{~m})$ and high gas flow rate (specific power $\varepsilon>0.1 \mathrm{~W} / \mathrm{kg}$ ). In general the effects of both the nozzle inclination and the nozzle location are larger in the shallow bath than in the deep bath.

The nozzle inclination and its location have no appreciable effect on the mixing time of the deep bath, while in the shallow bath it can be significantly improved by changing the nozzle inclination and its location. 


\section{Nomenclature}

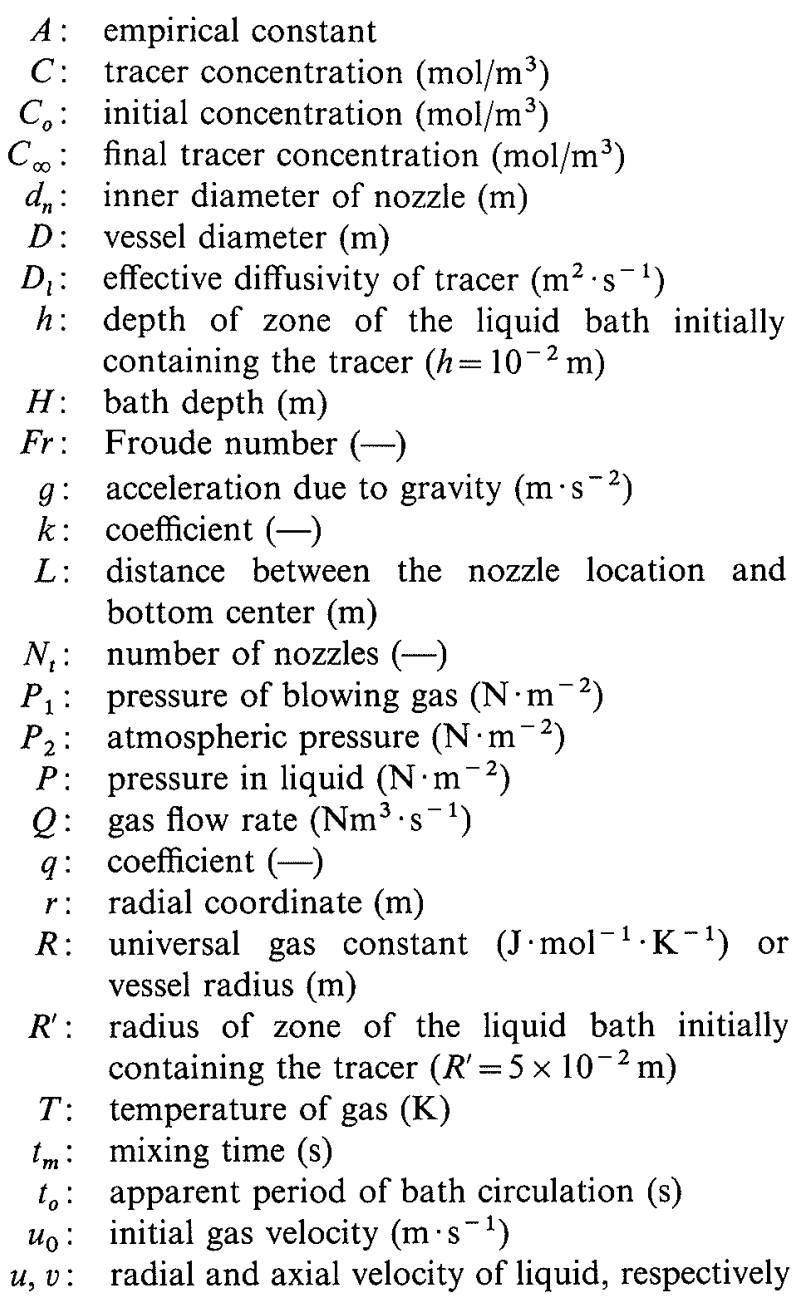

$\left(\mathrm{m} \cdot \mathrm{s}^{-1}\right)$

$v_{p}:$ average plume rise velocity $\left(\mathrm{m} \cdot \mathrm{s}^{-1}\right)$

$V, V_{\infty}$ : voltage changed with time and final voltage (V)

$V_{M}:$ molar volume of gas $\left(\mathrm{m}^{3} \cdot \mathrm{mol}^{-1}\right)$

$W:$ mass of liquid $(\mathrm{kg})$

$z:$ axial coordinate $(\mathrm{m})$

$\varepsilon:$ total specific power $\left(\mathrm{m}^{2} \cdot \mathrm{s}^{-3}\right)$

$\varepsilon_{b}:$ specific buoyancy power $\left(\mathrm{m}^{2} \cdot \mathrm{s}^{-3}\right)$

$\varepsilon_{j}:$ specific kinetic power $\left(\mathrm{m}^{2} \cdot \mathrm{s}^{-3}\right)$

$\mu_{1}$ : effective viscosity of liquid $\left(\mathrm{kg} \cdot \mathrm{m}^{-1} \cdot \mathrm{s}^{-1}\right)$

$\rho_{l}, \rho_{g}:$ density of liquid and gas, respectively $\left(\mathrm{kg} \cdot \mathrm{m}^{-3}\right)$ $\tau:$ time (s)

\section{REFERENCES}

1) K. Mori and M. Sano: Tetsu-to-Hagané, 6 (1981), 672.

2) O. Haida and J. K. Brimacombe: SCANINJECT III, Lulea, Sweden, (1983), 5: 1.

3) G. G. Krishna Murthy, S. P. Mehrotra and A. Ghosh: Metall. Trans., 19B (1988), 839.

4) J. D. Hwang, Y. C. Lin and W. S. Hwang: Mater. Sci. Technol,, 6 (1990), 376.

5) A. Murthy, J. Szekely and N. El-Kaddah: Metal. Trans., 19B (1988), 765 .

6) S. T. Johansen, D. G. C. Robertson, K. Woje and T. A. Engh: Metall. Trans., 19B (1988), 745.

7) V. I. Baptizmansky and Yu. S. Paniotov: Izv. V.U.Z. Chernaya Metall., 6 (1989), 26.

8) V. Sahajwalla, J. K. Brimacombe and M. E. Salcudean: Iron Steelmaker, 16 (1989), No. 10, 39.

9) D. Mazumdar: Metall. Trans., 20B (1989), 967.

10) J. Mietz and F. Oeters: Can. Metall. $Q$, 28 (1989), 19.

11) G. D. Byrne and C. A. Hall: Numerical Solution of System of Nonlinear Algebraic Equations, Academic Press, New York and London, (1973), 110. 